\title{
Cirugía de Switch arterial o corrección anatómica de la transposición de los grandes vasos Artícullo de revisión
}

Aguilar-Alaníz Elizabeth*, Reyes-Pavón Rodrigo**

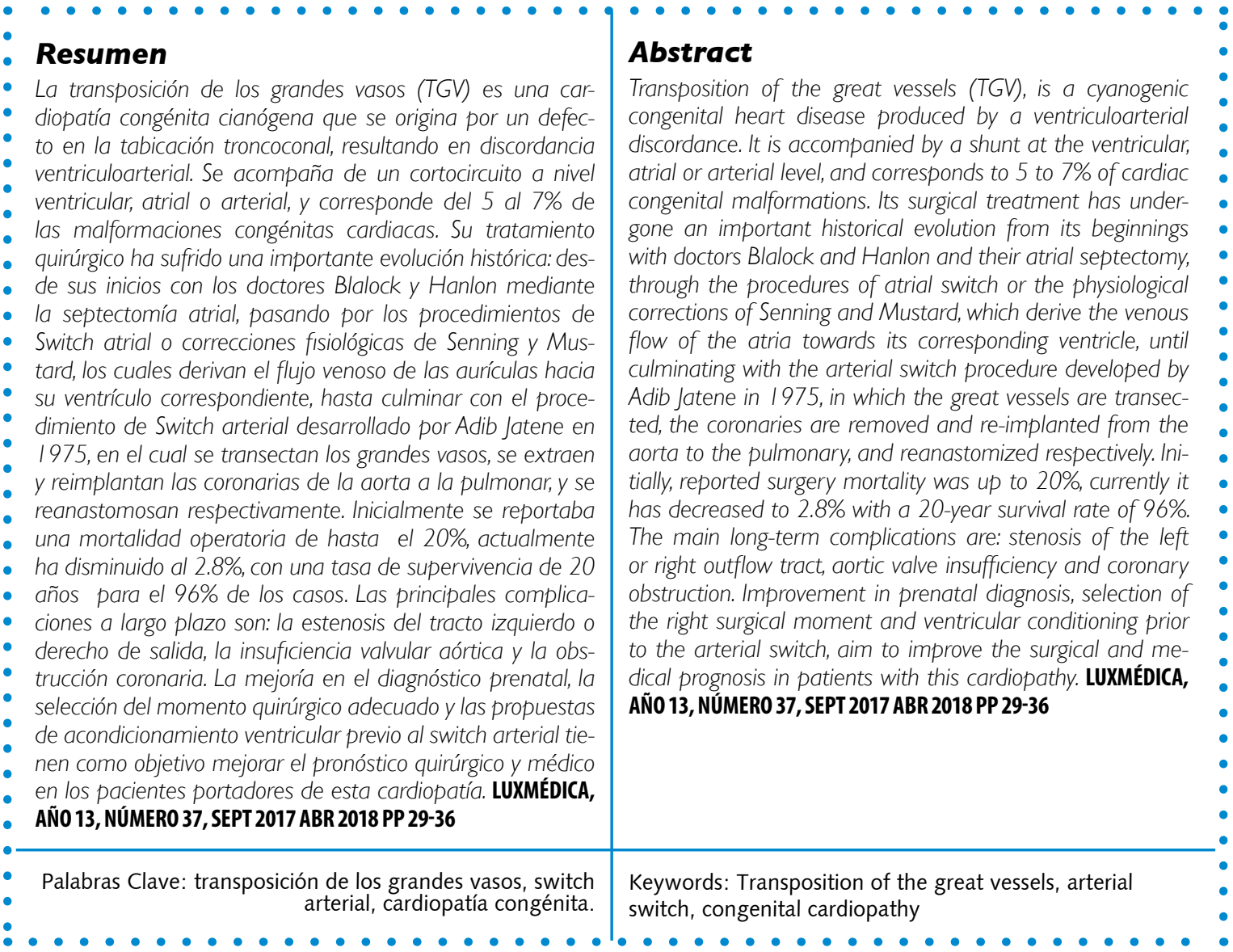

* Aguilar- Alaníz Elizabeth. Cirujano Cardiotorácico y de Malformaciones Congénitas. Jefe del Departamento de Cirugía Cardiovascular del Centenario Hospital Miguel Hidalgo. Correo electrónico eaguilar12598@gmail.com

** Reyes-Pavón Rodrigo. Estudiante del séptimo semestre de la carrera de Médico Cirujano del Centro de Ciencias de la Salud de la Universidad Autónoma de Aguascalientes. Correo electrónico rodrp1303@gmail.com

Fecha de recibido: 13 de noviembre 2017

Fecha de aceptación: 15 de enero 2018

Correspondencia: Dra. Elizabeth Aguilar Alaníz. Departamento de Cirugía Cardiovascular, tercer piso. Centenario Hospital Miguel Hidalgo. Galeana sur no. 465, colonia Obraje, código postal 20230, Aguascalientes, Ags. México.Teléfono: 014499946720 extensión 4513. Correo electrónico: eaguilar12598@gmail.com 


\section{Introducción}

La transposición de los grandes vasos (TGV), descrita inicialmente por Baillie en 1797, es una cardiopatía congénita cianógena que se origina por un defecto en la tabicación troncoconal, resultando en discordancia ventriculoarterial; es decir, una aorta que emerge del ventrículo derecho y se posiciona anterior y a la derecha, y una arteria pulmonar que surge del ventrículo izquierdo y es posterior y paralela a la aorta. Para ser compatible con la vida, debe acompañarse de un cortocircuito a nivel ventricular, atrial o arterial, siendo el primero el más frecuente, presente en casi la mitad de los casos. ${ }^{1}$ Corresponde del 5 al 7\% de las malformaciones congénitas cardiacas, con una prevalencia de 0.2 por cada 1000 nacidos vivos, teniendo predominio en el sexo masculino. ${ }^{2}$

El tratamiento quirúrgico tiene sus inicios en el Hospital Johns Hopkins en 1950, con Alfred Blalock y Rollins Hanlon mediante la septectomía atrial; luego, en 1966, Rashkind y Miller describen la atrioseptostomía con balón, un procedimiento menos riesgoso aunque ambos métodos únicamente son paliativos. Baffes, en 1955, realiza el primer switch venoso, dirigiendo la vena cava inferior con un injerto aórtico homólogo al atrio izquierdo, y las venas pulmonares derechas al atrio derecho.

En 1957, Ake Senning realiza exitosamente el primer switch atrial usando colgajos atriales en un niño de 9 meses. Mustard, en 1963, opera a una niña de 18 meses reparando el defecto septal ventricular y realizando el switch atrial con pericardio autólogo, basándose en la técnica propuesta en 1955 por el doctor Harold Albert, consistente en el uso de pericardio para formar barreras y derivar el flujo de las venas cavas hacia la válvula mitral y de las venas pulmonares hacia la válvula tricúspide. Sin embargo, se reportaron obstrucciones y ausencia de crecimiento de las barreras. De esta manera, se originan los dos procedimientos de corrección fisiológica o de switch atrial. ${ }^{1,3}$ Es en 1975, cuando el cirujano brasileño Adib Jatene publica el primer caso exitoso de corrección anatómica o Cirugía de Switch Arterial (CSA) en un lactante menor de 40 días de nacido con TGV asociada a Comunicación Interventricular (CIV). La técnica quirúrgica que describe, refiere que usando hipotermia profunda y paro cardíaco se liberan las coronarias con un botón de la pared aórtica, se cierra la pared aórtica con parche de duramadre homóloga, y se re-implantan las coronarias en la arteria pulmonar. Luego, se lleva a cabo la transección de la aorta ascendente y el tronco de la pulmonar, se contraponen y se reanastomosan. Finalmente, a través de ventriculotomía derecha se cierra el defecto septal interventricular con un parche de Dacron y se sutura mediante puntos interrumpidos con mersilene. El estudio hemodinámico postquirúrgico mostró corrección total, el paciente fue egresado a las tres semanas y evolucionó sin complicaciones. ${ }^{4}$ 
Dicha técnica ha sufrido importantes modificaciones, entre ellas la maniobra de translocación de la pulmonar anterior a la aorta para reducir la tensión y eliminar la necesidad de injerto, descrita por Lecompte en 1981.5 Williams y cols. sugieren reconstruir el tracto de salida del ventrículo derecho con un injerto tubular, conectando la aorta proximal a la bifurcación de la arteria pulmonar, ya que disminuye la obstrucción generada por la tensión de la anastomosis sin injerto. ${ }^{6}$

En México, de acuerdo al Primer Informe de Cirugía Cardíaca Pediátrica, de 2011 a 2012 se registraron 943 procedimientos quirúrgicos, de los cuales 27 (2.8\%) fueron procedimientos de Jatene $y$ de estos, 13 (48\%) fueron asociados a cierre de CIV. ${ }^{7}$

El presente artículo tiene como objetivo llevar a cabo una revisión de los aspectos básicos de los abordajes para la corrección quirúrgica de la TGV, así como analizar los resultados postquirúrgicos de la corrección anatómica (procedimiento de Jatene) en la literatura.

\section{Reparación quirúrgica de la transposición de los grandes vasos}

Existen dos tipos de reparación quirúrgica para los pacientes con TGV: una es la re- paración fisiológica (figura 1), consistente en la derivación del flujo arterial o venoso mediante septos intraatriales hacia el ventrículo correspondiente, conservando al ventrículo derecho como sistémico y al izquierdo como venoso. Otra es la reparación anatómica actual (figuras 2 y 3), que consiste en cerrar mediante atriotomía derecha la CIV si está presente, realizar la transección de los grandes vasos y la resección de los ostia coronarios que se anastomosan a la neoaorta (arteria pulmonar nativa), llevar a cabo la maniobra de Lecompte para traer la bifurcación de las arterias pulmonares anterior a la aorta $y$, posteriormente, la reconstrucción de las nuevas arterias. En el caso de la neopulmonar (arteria aorta nativa), se reconstruye mediante un injerto de pericardio ya sea autólogo o heterólogo; y en el caso de la neoaorta, la reconstrucción se realiza con la implantación de los ostia coronarios. Finalmente, se reanastomosan los vasos correspondientes: la aorta distal con el vaso en el que se implantaron las coronarias y la pulmonar distal con el vaso del cual se resecaron los ostias coronarios $y$ en el que se repararon los defectos con el injerto de pericardio. De esta manera, se restaura la anatomía y fisiología normal del corazón, relacionando cada ventrículo con su arteria correspondiente. ${ }^{10}$

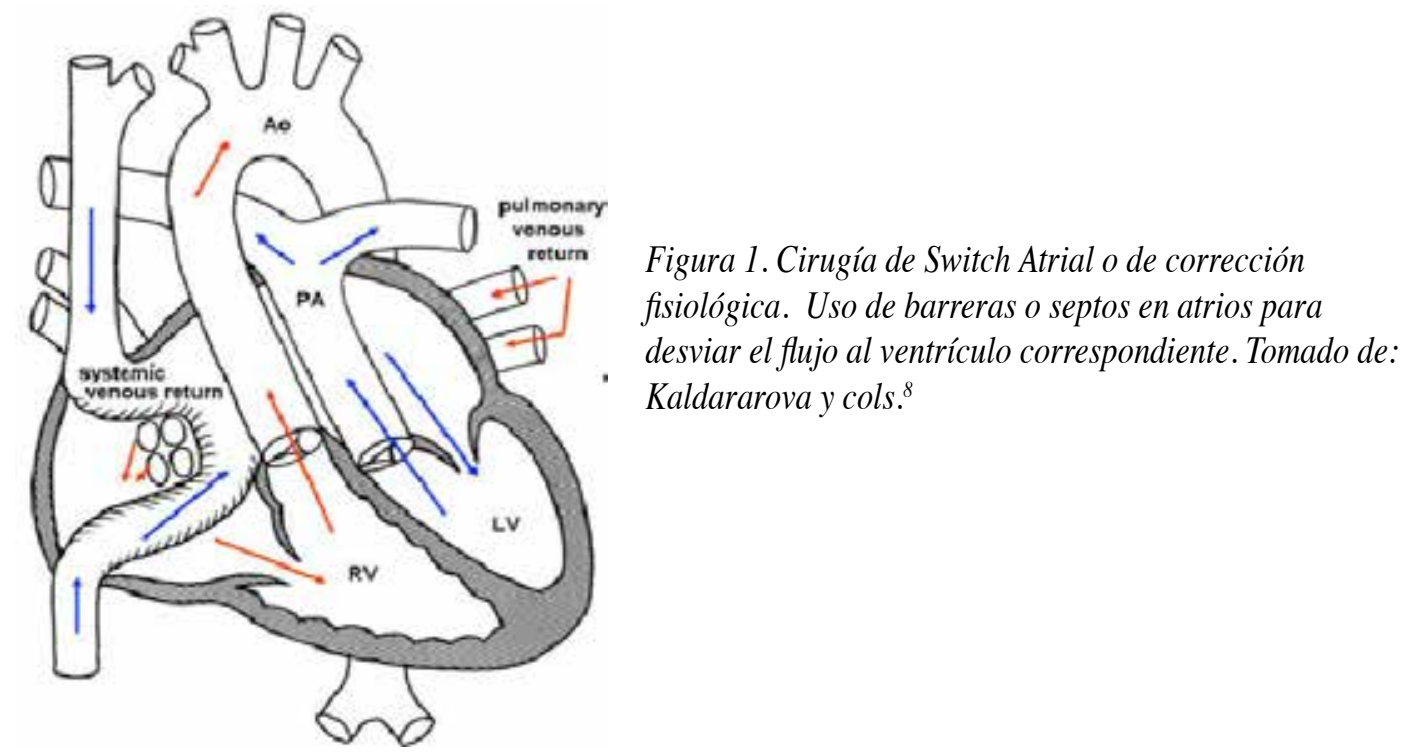

Figura 1. Cirugía de Switch Atrial o de corrección fisiológica. Uso de barreras o septos en atrios para desviar el flujo al ventrículo correspondiente. Tomado de: Kaldararova y cols. ${ }^{8}$ 


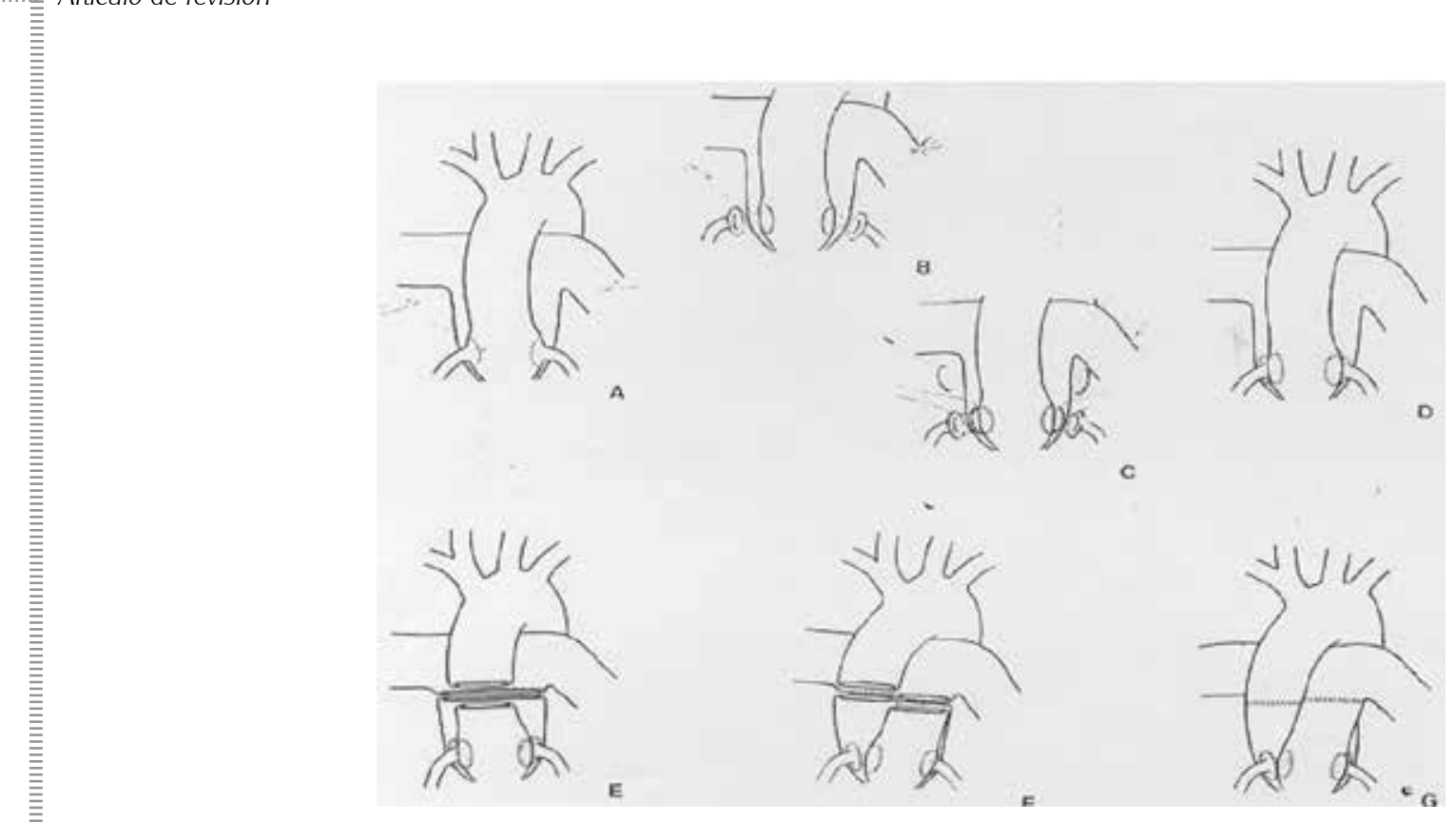

Figura 2. Diagrama original de Jatene demostrando procedimiento para Switch Atrial. Tomado de: Jatene y cols. ${ }^{4}$
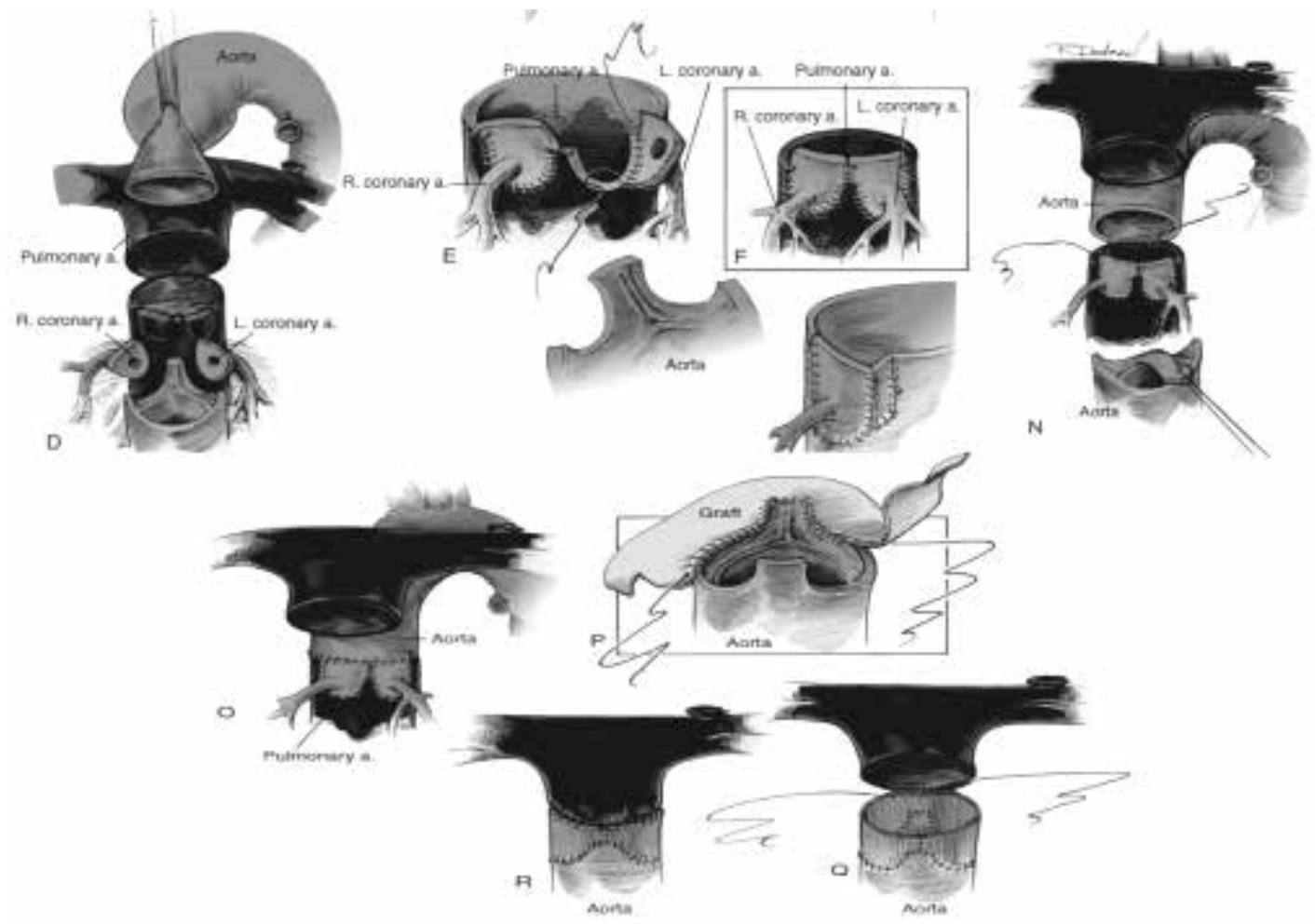

Figura 3. Cirugía de Switch arterial en la actualidad.

$D$ : Transección de los grandes vasos. E, $F, N, O$ : resección e implantación de los ostia coronarios en la neoaorta. P: reconstrucción de la neopulmonar con injerto. $Q$ y $R$ : anastomosis de neopulmonar. Tomado de: Sellke y cols. ${ }^{9}$ 
La principal desventaja de los procedimientos de corrección fisiológica es la presencia de arritmias crónicas, ocasionadas por la manipulación atrial que resulta en daño al nodo sinusal y al tejido de conducción, con probabilidad potencial de muerte súbita (más frecuente en el procedimiento de Senning) y de insuficiencia cardíaca (más frecuente en el procedimiento de Mustard). Ésta última, además, se ve agravada por el hecho de conservar al ventrículo derecho como sistémico, y el consecuente desarrollo de insuficiencia tricuspídea. Se ha documentado que la supervivencia a 20 años de estos procedimientos es del $78 \%$ para el Mustard y $84 \%$ para el Senning, mientras que para la CSA es de casi el $97 \%$, por ello, se prefiere siempre que sea posible. ${ }^{11}$
En uno de los primeros reportes de seguimiento a largo plazo del procedimiento de Jatene realizado en 184 pacientes, se reportaba una mortalidad posquirúrgica inmediata del $20.7 \%$ y del $2.7 \%$ en los siguientes 9 a 66 meses del seguimiento. ${ }^{12}$ Actualmente, la mortalidad operatoria es cercana al $2.8 \%$ debido a complicaciones coronarias; la tasa de supervivencia a 20 años es del $96 \%$; menos del $20 \%$ de los pacientes requieren de reoperación tardía secundaria a estenosis del tracto derecho o izquierdo de salida, insuficiencia valvular aórtica u obstrucción coronaria (asintomática o no). ${ }^{13,14,15}$

En la Tabla 1 se reportan los resultados de morbilidad y mortalidad posquirúrgica obtenidos en diferentes estudios retrospectivos y de seguimiento.

\section{Tabla I}

Morbimortalidad posquirúrgica después de la cirugía de Switch arterial. NR: No reporta. NYHA: New York Heart Association. Bloqueo AV: Bloqueo Auriculoventricular.

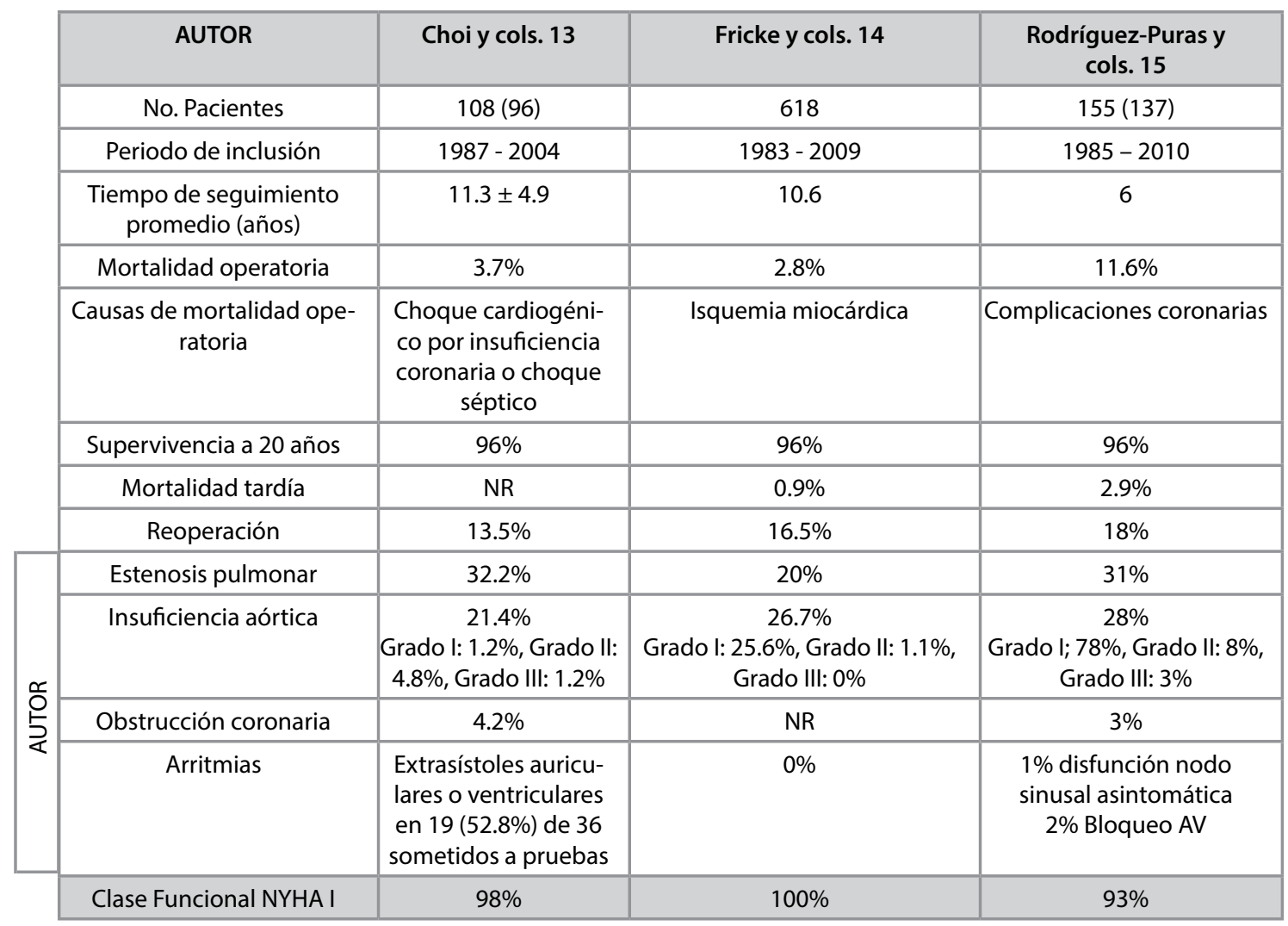


En el Instituto Nacional de Cardiología "Ignacio Chávez", de 1991 a 2003, se reportaron 91 cirugías de Switch Arterial, con una mortalidad global del $20 \%$, inicialmente fue del $52 \%$ y en los últimos 5 años hubo un descenso a menos del $10 \%$, utilizando preparación del ventrículo izquierdo (mediante bandaje pulmonar, véase más adelante) en pacientes con septo íntegro y mayores a 3 semanas de vida. ${ }^{16}$

La decisión del momento quirúrgico también ha cambiado conforme mejoraron los conocimientos de la fisiología en el corazón con TGV, ya que se observó que, después del primer mes de vida, el ventrículo izquierdo, especialmente en los casos sin CIV, tenía dificultades para tolerar la poscarga de la circulación sistémica.

Baño-Rodrigo y cols. encontraron que, después del nacimiento, y conforme pasa el tiempo y las resistencias vasculares pulmonares disminuyen, ocurre únicamente una sobrecarga volumétrica en el ventrículo izquierdo con septo íntegro, por lo que hay poco crecimiento e hipertrofia del mismo en comparación con el ventrículo derecho. En caso de que haya un defecto interventricular, el ventrículo izquierdo encuentra una sobrecarga de volumen y también de presión, por lo que su crecimiento es mayor, pero aún por debajo de lo normal. El menor espesor de la pared libre, la masa ventricular disminuida y la geometría anormal de la cámara en el ventrículo izquierdo, así como las alteraciones en la contracción septal y el mayor grosor en el ventrículo derecho, son causas por las cuales el ventrículo izquierdo puede ser incapaz de asumir la poscarga sistémica después de la corrección anatómica. ${ }^{17}$

Por lo antes expuesto, se ha considerado que el ventrículo izquierdo es capaz de tolerar la presión sistémica, si se realiza la corrección antes de los 21 días de nacimiento. Si no es así, puede llevarse a cabo la corrección en dos etapas: en una etapa inicial, el bandaje de la arteria pulmonar (acompañado o no de fístula sistémico-pulmonar) para re-acondicionar al ventrículo izquierdo; y en la segunda, la CSA definitiva en los siguientes siete días, con resultados satisfactorios. ${ }^{18,19}$ Esto presenta utilidad cuando el diagnóstico se retrasa o la cirugía no puede llevarse a cabo en el momento idóneo. Las razones para tal retraso se generan por la falta de diagnóstico prenatal sistemático, la demora de la detección posnatal y enfermedades e infecciones mayores cuyo tratamiento posterga la cirugía, esto es especialmente común en países en desarrollo. Schidlow y cols. demostraron que, de 778 procedimientos de Jatene llevados a cabo de 2010 a 2013 en 15 países diferentes en vías de desarrollo, solo el $11 \%$ se realizó en la primera semana de vida, mientras que el $49 \%$ se realizó en el primer mes de vida, el $45 \%$ entre el primer mes y el primer año, y el restante $6 \%$ entre el primer y el decimosegundo años de vida. ${ }^{20}$

Algunas revisiones de dicho abordaje en dos etapas, sin embargo, han reportado: tasas de mortalidad mayores, tiempos de estancia en la Unidad de Terapia Intensiva prolongados, y mayor incidencia de disfunción ventricular tardía, insuficiencia neoartica y obstrucción del tracto derecho de salida. ${ }^{21}$ A pesar de esto, se prefiere dicho abordaje sobre los procedimientos de corrección fisiológica, por las complicaciones de los mismos a largo plazo ya mencionadas. Por este motivo, se ha intentado realizar la CSA primaria en TGV con septo íntegro después de los primeros 21 días, con evidencia de éxito en lactantes mayores a 6 semanas, mediante el uso de Oxigenación por Membrana Extracorpórea (ECMO) con circuito integrado en aquellos con geometría ventricular izquierda desfavorable (definida como ventrículo izquierdo con forma ecográfica de "banana") y alta predictibilidad de necesidad de apoyo circulatorio mecánico posquirúrgico. El ECMO, además de permitir el acondicionamiento ventricular bajo normoxemia y control en la precarga, disminuye probabilidades de paro cardiaco, y estado de bajo gasto cardiaco. ${ }^{22}$ 
En algunos pacientes con hipertensión pulmonar asociada, el ventrículo izquierdo puede crecer y mantener su capacidad de soportar la presión sistémica pasadas las primeras tres semanas de vida. Liu y cols. realizaron un estudio en pacientes mayores de seis meses de edad con TGV asociada a CIV no restrictiva e hipertensión pulmonar severa reversible sometidos a corrección anatómica por CSA y concluyeron que los resultados a corto y mediano plazo (en un seguimiento por 42 meses) eran excelentes, con una mortalidad temprana del $7 \%$ y una tasa de supervivencia del $97.2 \%$. De los supervivientes, el $97.2 \%$ se encontraban en clase funcional de la New York Heart Association (NYHA) I, solo un 2.9\% tenía insuficiencia aórtica, otras complicaciones se encontraron en menos del $6 \%$, y la media de la presión pulmonar media fue de $22.4 \pm 8.7 \mathrm{mmHg} .{ }^{23}$

La decisión quirúrgica entre CSA en una o dos etapas puede realizarse con los criterios ultrasonográficos que incluyen el movimiento septal paradójico, morfología ventricular invertida y adelgazamiento de la pared libre del ventrículo. En caso de estar presentes dichos criterios, puede optarse por la corrección anatómica en dos etapas. ${ }^{24,} 25$ Para evaluar el adecuado preacondicionamiento ventricular se sugiere utilizar los criterios ultrasonográficos de presión ventricular izquierda del $90 \%$ de la presión sistémica, fracción de eyección $>0.55$, presión telediastólica $<12 \mathrm{mmHg}$, y una masa ventricular izquierda de $65 \mathrm{~g} /$ $\mathrm{m}^{2}$ obtenida por resonancia magnética. ${ }^{25}$

Alternativamente se ha sugerido realizar una prueba de bandaje de la arteria pulmonar, aumentando la presión del ventrículo izquierdo hasta un nivel de presión sistémica (índice de presión pulmonar/ presión sistémica o $\mathrm{P} / \mathrm{s}$ de 0.8 a 1.0) durante 15 a 30 min, si posterior a esto no hay datos de falla ventricular izquierda, la CSA puede realizarse en una etapa. ${ }^{26}$ En el caso de TGV asociada a CIV, el retraso en el momento quirúrgico tiene el riesgo de insuficiencia cardiaca congestiva y desarrollo temprano de vasculopatía pulmonar obstructiva, además de que la CIV puede disminuir espontáneamente resultando en presiones ventriculares izquierdas pulmonares y la consecuente regresión ventricular a una posición subpulmonar al momento de la corrección, por esto, se recomienda su corrección a la edad más temprana posible. ${ }^{27}$

\section{Conclusiones}

El tratamiento quirúrgico de la TGV ha sufrido una importante evolución histórica y múltiples contribuciones técnicas hasta culminar con la CSA, que ha representado la mejor opción para corregir su anatomía, y restaurar la fisiología circulatoria normal. Su popularidad ha resultado en una disminución de la morbimortalidad en etapas posquirúrgicas tanto inmediatas como tardías. Nuestro país ha tratado de adoptar dicho abordaje quirúrgico, pero debido al diagnóstico y referencia tardíos, la cirugía de switch atrial y la corrección en dos etapas aún juegan un papel importante, a pesar de las complicaciones y desenlaces adversos inherentes a las técnicas. Por esta razón, las propuestas de regionalización de recursos materiales y profesionales que incluyen, entre otras cosas, un aumento, mejora y oportunidad en el diagnóstico prenatal, atención hospitalaria de manera pronta, así como la selección del momento quirúrgico adecuado, tienen como objetivo mejorar el resultado en los pacientes portadores de esta cardiopatía. 


\section{Bibliografía}

1. Nagre SW. Historical evolution of surgery for transposition of great arteries (TGA). J Cardiovasc Med Cardiol. 2016; 3: 026-029.

2. Villafañe J. Lantin-Hermoso M.R. Bhatt A.B. et al. DTransposition of the great arteries, the current era of the arterial switch operation. J Am Coll Cardiol. 2014; 64: 498-511.

3. Marathe S.P. Talwari S. Surgery for transposition of great arteries: a historical perspective. Ann Pediatr Cardiol. 2015; 8: 122-128.

4. Jatene A.D. Fontes V.F. Paulista P.P. et al. Successful anatomic correction of transposition of the great vessels, a preliminary report. Arq Bras Cardiol. 1975; 28: 461-464.

5. Lecompte Y. Zannini L. Hazan E. et al. Anatomic correction of transposition of the great arteries. J Thorac Cardiovasc Surg. 1981; 82: 629-631.

6. Williams W.G. Freedom R.M. Culham G. et al. Early experience with arterial repair of transposition. Ann Thorac Surg. 1981; 32: 8-15.

7. Cervantes J.L. Calderón J. Ramírez S. et al. El registro mexicano de cirugía cardiaca pediátrica. Primer informe. Rev Invest Clin. 2013; 65: 476-482.

8. Kaldararova M. Balazova E. Bordacova L. et al. Arrhythmias in congenital heart defects. Bratisl Lek Listy. 2007; 108: 14-19.

9. Pigula F.A. Del Nido P.J. Transposition of the great arteries: simple and complex forms. En: Sellke F.W. Del Nido P.J. Swanson S.J. Sabiston \& spencer: surgery of the chest. 9a edición. Philadelphia, Elsevier, 2016, pag. 2243-2266.

10. Ároca A. Cirugía de la transposición de las grandes arterias con septo ventricular íntegro. Cir Cardiov. 2008; 15: 345-349.

11. Raissadati A. Nieminen H. Sairanen H. Jokinen E. Outcomes after the mustard, senning and arterial switch operation for treatment of transposition of the great arteries in finland: a nationwide 4-decade perspective. Eur J Cardiothorac Surg. 2017; 52: 573580.

12. Jatene F.B. Bosisio I.B.J. Jatene M.B. Souza L.C.B. Barbero-Martial M. Jatene A.D. Late results (50 to 182 months) of the jatene operation. Eur J Cardiothorac Surg. 1992; 6: 575-578.

13. Choi B.S. Kwon B.S. Kim G.B. et al. Long-term outcomes after an arterial switch operation for simple complete transposition of the great arteries. Korean Circ J. 2010; 40: 23-30.

14. Fricke T.A. d'Udekem Y. Richardson M. et al. Outcomes of the arterial switch operation for transposition of the great arteries: 25 years of experience. Ann Thorac Surg. 2012; 94: 139-145.

15. Rodríguez-Puras M.J. Cabeza-Letra L. Romero-Vazquianez $M$. et al. Mid-term morbidity and mortality of patients after arterial switch operation in infancy for transposition of the great arteries. Rev Esp Cardiol. 2014; 67:181-188.

16. Ramírez S. Cervantes J.L. Transposición de grandes arterias. Resultados de la corrección anatómica en el Instituto Nacional de Cardiología "Ignacio Chávez". Arch Cardiol Méx. 2004; 74: S326-S329.

17. Baño-Rodrigo A. Quero-Jiminez M. Moreno-Granado F. Gamallo-Amat C. Wall thickness of ventricular chambers in transposition of the great arteries: surgical implications. J Thorac Cardiovasc Surg.1980; 79: 592- 597.

18. Yacoub M.H. Radley-Smith R. Maclaurin R. Two-stage operation for anatomical correction of transposition of the great arteries with intact interventricular septum. Lancet. 1977; 1: 1275-1278.

19. Jonas R.A. Giglia T.M. Sanders S.P. et al. Rapid, twostage arterial switch for transposition of the great arteries and intact ventricular septum beyond the neonatal period. Circulation. 1989; 80: 1203 - 1208.

20. Schidlow D. Jenkins K. Gauvreau K. et al. Transposition of the great arteries in the developing world surgery and outcomes. J Am Coll Cardiol. 2017; 69 43-51.

21. Ismail. S.R. Kabbani S. Najm H.K. Abusuliman R.M Elbarbary M. Early outcome for the primary arterial switch operation beyond the age of 3 weeks. Pediatr Cardiol. 2010; 31: 663-667.

22. Bisoi A.K. Sharma P. Chauhan S. et al. Primary arterial switch operation in children presenting late with $\mathrm{d}$-transposition of great arteries and intact ventricular septum. When is it too late for a primary arterial switch operation? Eur J Cardiothorac Surg. 2010; 38 $707-713$

23. Liu Y.L. Hu S.S. Shen X.D. et al. Midterm results of arterial switch operation in older patients with severe pulmonary hypertension. Ann Thorac Surg. 2010; 90: $848-855$.

24. Gil-Jaurena J.M. Castillo R. Zabala J.I. Conejo L. Cuenca V. Picazo B. Late arterial switch, without previous preparation or extracorporeal membrane oxygenation back-up. Rev Esp Cardiol. 2012; 65: 1134-1142.

25. Watanabe N. Mainwaring R.D. Carrillo S.A. et al Left ventricular retraining and late arterial switch for d-transposition of the great arteries. Ann Thorac Surg. 2015; 99: 1655-1661.

26. Dabritz S. Engelhardt W. Bernuth G. Messmer B.J Trial of pulmonary artery banding: a diagnostic criterion for 'one-stage' arterial switch in simple transposition of the great arteries beyond the neonatal period. Eur J Cardiothorac Surg. 1997; 11: 112-116.

27. Wetter J. Belli E. Sinzobahamvya N. Blaschzok H.C. Brecher A.M. Urban A.E. Transposition of the great arteries associated with ventricular septal defect: surgical results and long-term outcome. Eur J Cardiothorac Surg. 2001; 20: 816-823. 